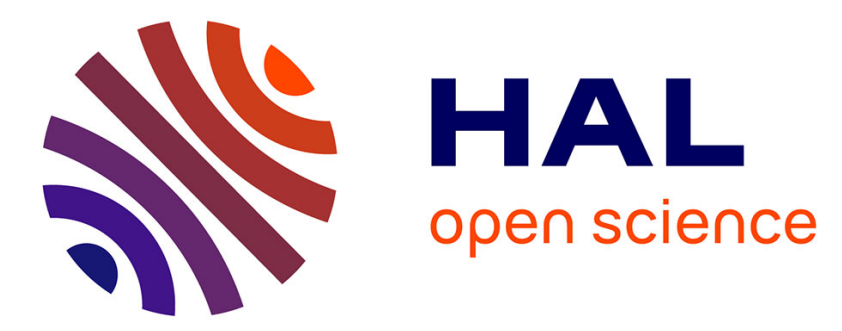

\title{
Performance of a low noise readout ASIC for the W-Si calorimeter physics prototype for the future linear collider
}

\author{
J. Fleury, C. de La Taille, G. Martin-Chassard
}

\section{To cite this version:}

J. Fleury, C. de La Taille, G. Martin-Chassard. Performance of a low noise readout ASIC for the W-Si calorimeter physics prototype for the future linear collider. International Conference on Calorimetry in High Energy Physics - CALOR2004 11, Mar 2004, Perugia, Italy. pp.191-195. in2p3-00022000

\section{HAL Id: in2p3-00022000 https://hal.in2p3.fr/in2p3-00022000}

Submitted on 30 Jun 2004

HAL is a multi-disciplinary open access archive for the deposit and dissemination of scientific research documents, whether they are published or not. The documents may come from teaching and research institutions in France or abroad, or from public or private research centers.
L'archive ouverte pluridisciplinaire HAL, est destinée au dépôt et à la diffusion de documents scientifiques de niveau recherche, publiés ou non, émanant des établissements d'enseignement et de recherche français ou étrangers, des laboratoires publics ou privés. 
Introduction: $\mathcal{F} \mathcal{L C}$ challenges for electronics

$C A L I C E=\mathcal{W}-S$ i Calorime ter

- Precision measurements : 10\%/vE

- good line arity (\%o level)

- Good inter-calibration (\% level)

- Lowcrosstalk (\%o level)

- Large dynamic range

- $0.1 \mathcal{M I P}->2500 \mathcal{M I P S}=15$ bits

- Lownoise

- Auto-trigger on $\mathcal{M I P}_{(40,000 \text { e-) }}$

- Hermeticity : no room for electronics!

- High level of integration : "SoC"

- Ultra-low power : (《mW/ch)

- 30 Mchannels

"Tracker electronics with calorimetric performance"

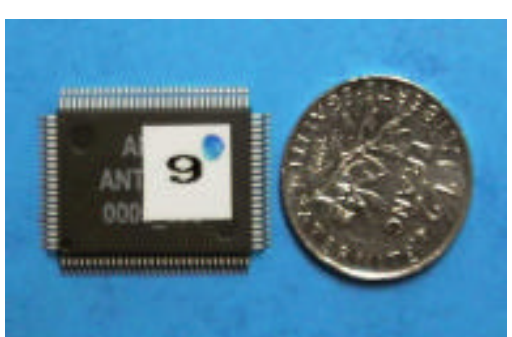

FLC 128ch 30*20mm $1 \mathrm{~W}$ ?
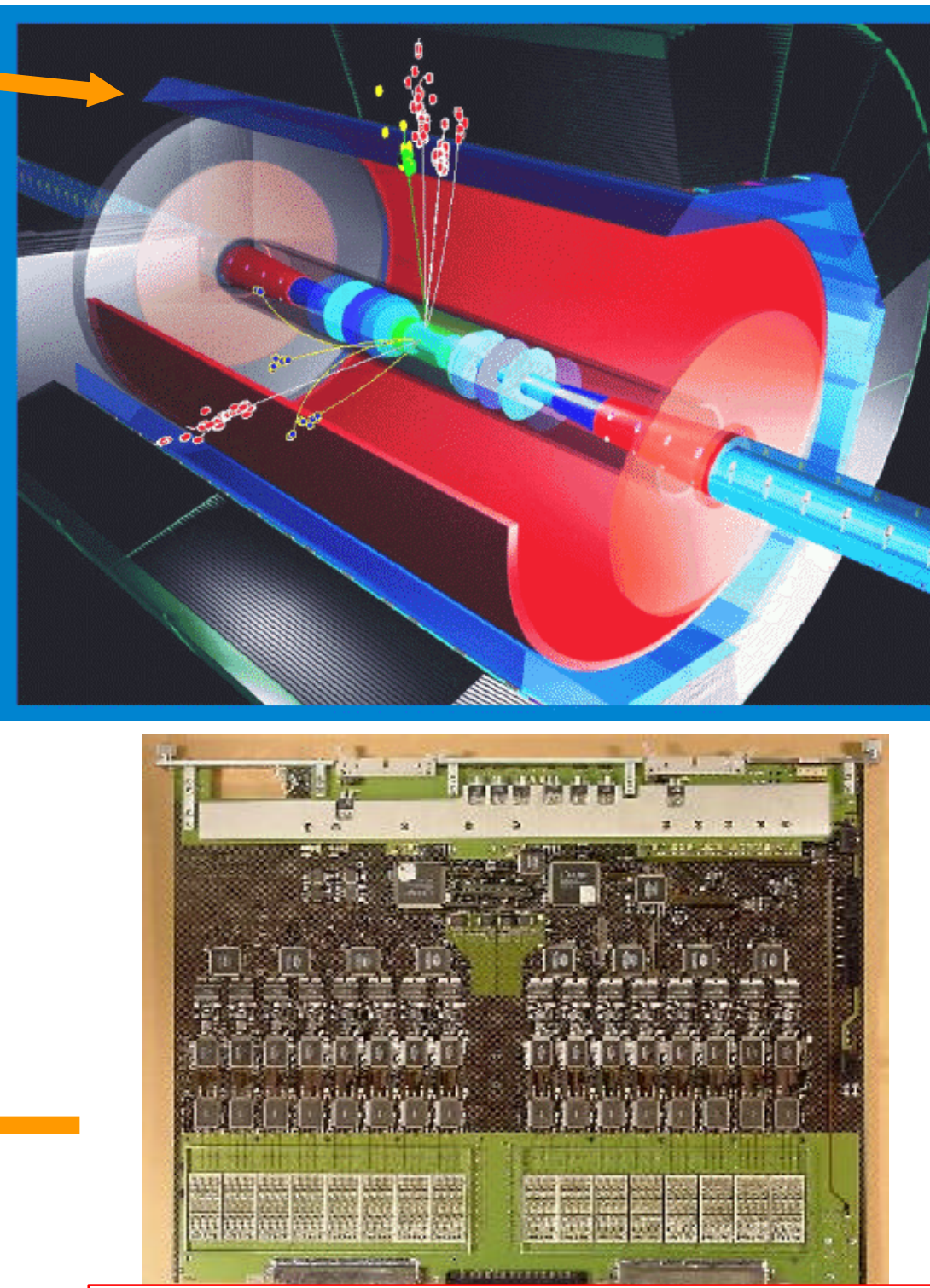

ATLAS LAr FEB 128ch 400*500mm $100 \mathrm{~W}$ 
- Multi-layer (30) W-Si prototype

- Active area: $18 \times 18 \mathrm{~cm}^{2}$, depth:24 $x_{0}$

- 30 detector slabs slid into alve olar structure

- See talkby g. Brient
Structure 2.8 ( $2 \times 1.4 \mathrm{~mm}$ of $\mathrm{W}$ plates)
Structure 1.4

(1.4mm of $\mathrm{W}$ plates)

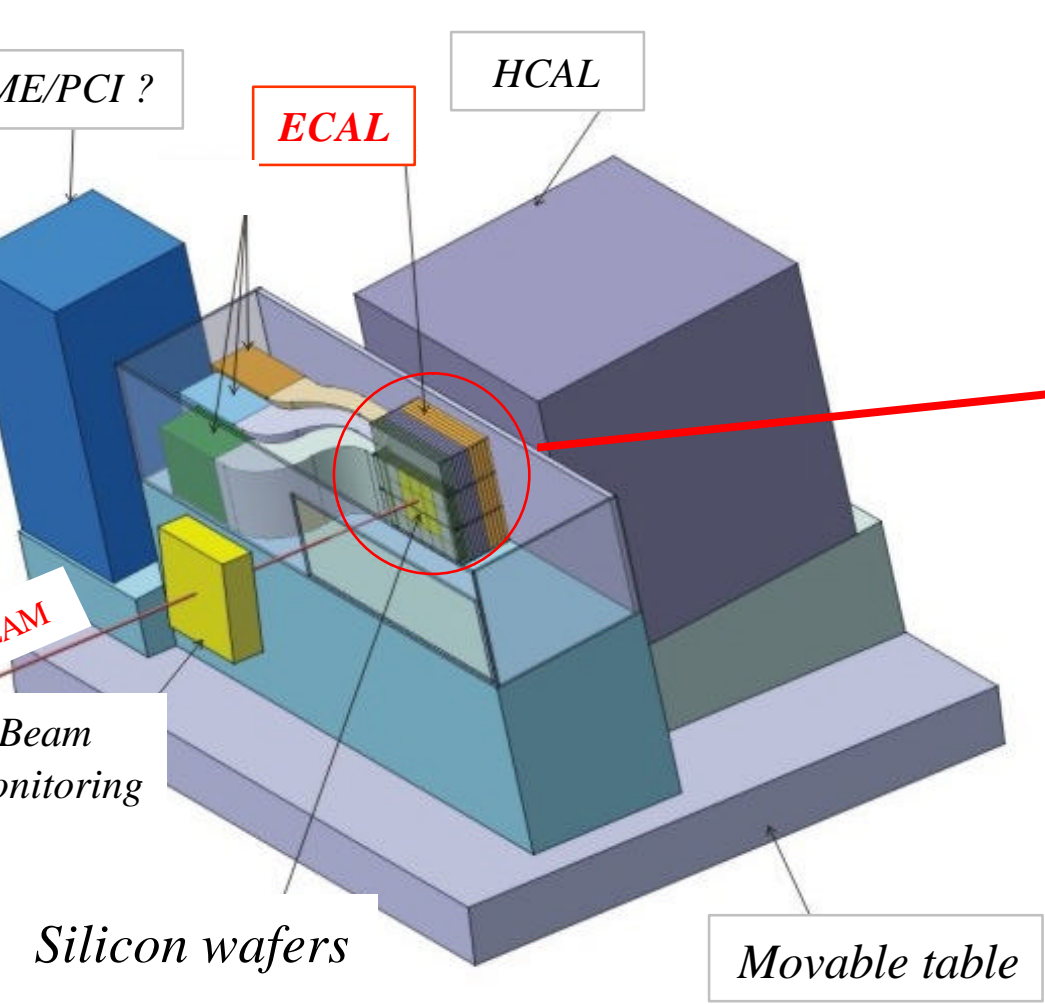

Structure 4.6 ( $3 \times 1.4 \mathrm{~mm}$ of W plates)

Metal inserts (interface)
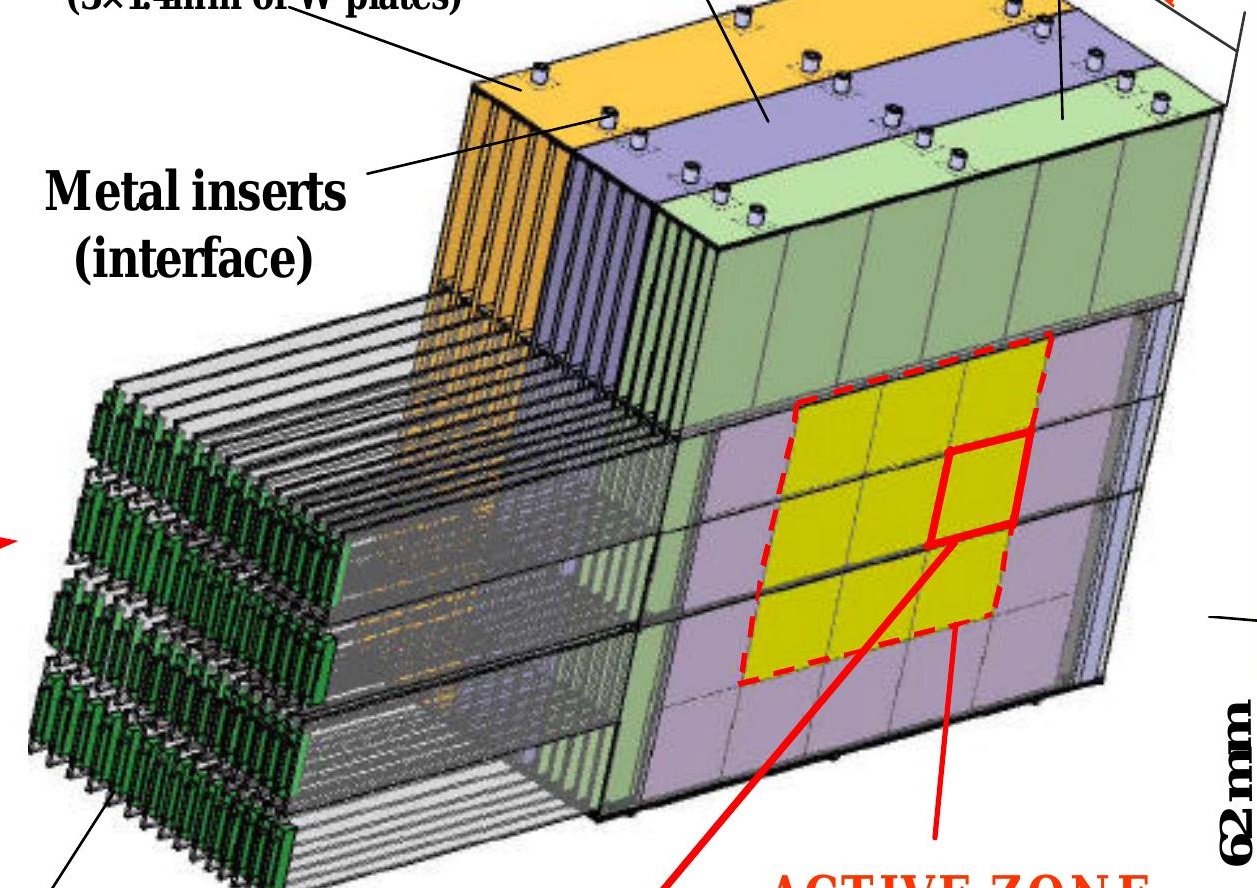

Detector slab (30)

ACTIVE ZONE $\left(18 \times 18 \mathrm{~cm}^{2}\right)$

Si wafer $6 \times 6$ diodes 
- Matrix of $6 \times 6$ pixels of $1 \mathrm{~cm}^{2}$

- Lowcost $=>$ simple process

- 2 manufacturers:

- IN(P Moscow

- Institute of Physics Prague

- AC coupling on $P C B$

4" High resistive wafer : $5 \mathrm{~K} \Omega \mathrm{cm}$

Thickness : 525 microns $\pm 3 \%$

Tile side : $62.0^{+0.1}{ }_{-0} \mathrm{~mm}$

\section{Guard ring}

In Silicone $~ 80$ e-h pairs / micron $\Rightarrow 42000 \mathrm{e}^{-} / \mathrm{MiP}$

Capacitance : $\sim 25 \mathrm{pF}$

Leakage current : 1 - $5 \mathrm{nA}$

Full depletion bias : $\sim 150 \mathrm{~V}$

Nominal operating bias : $200 \mathrm{~V}$
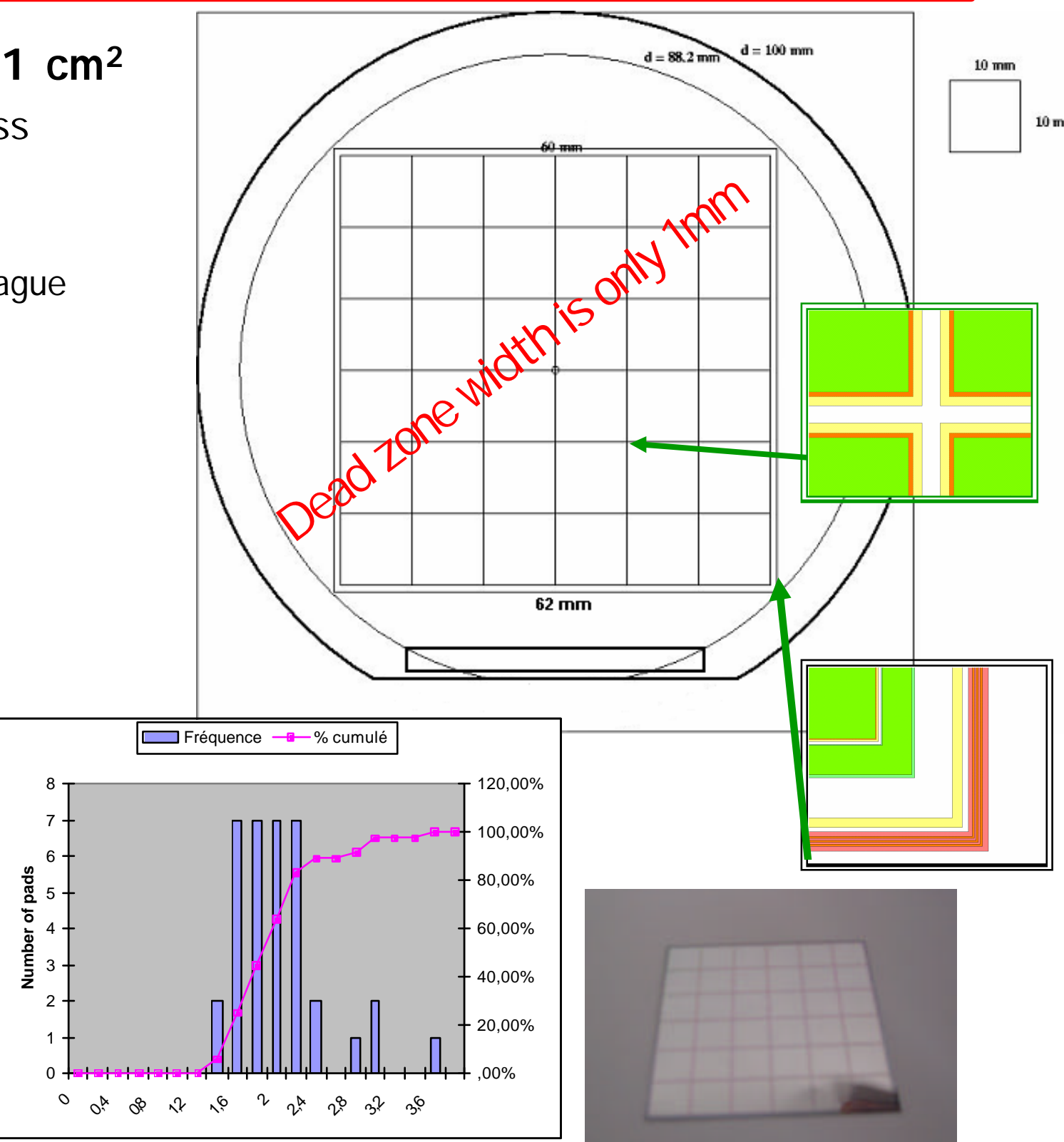
6 active wafers

Made of 36 silic on PIN diodes 216 channels per 6 oard Each diode is a $1 \mathrm{~cm}^{2}$ square
2 calibration switches chips

6 calibration channels per chip 18 diodes per calibration channel See talk on $\mathcal{A} \mathcal{T} \mathcal{A} \mathcal{A}$ calibration
$12 \mathcal{F L C P H} 3$ front-end chip

18 channels per chip

13 bit dynamic range
Line buffers To $\mathcal{D A Q}$ part Differential

\section{4 layers $2.1 \mathrm{~mm}$ thick Made in korea}


Front-end electronics synoptic

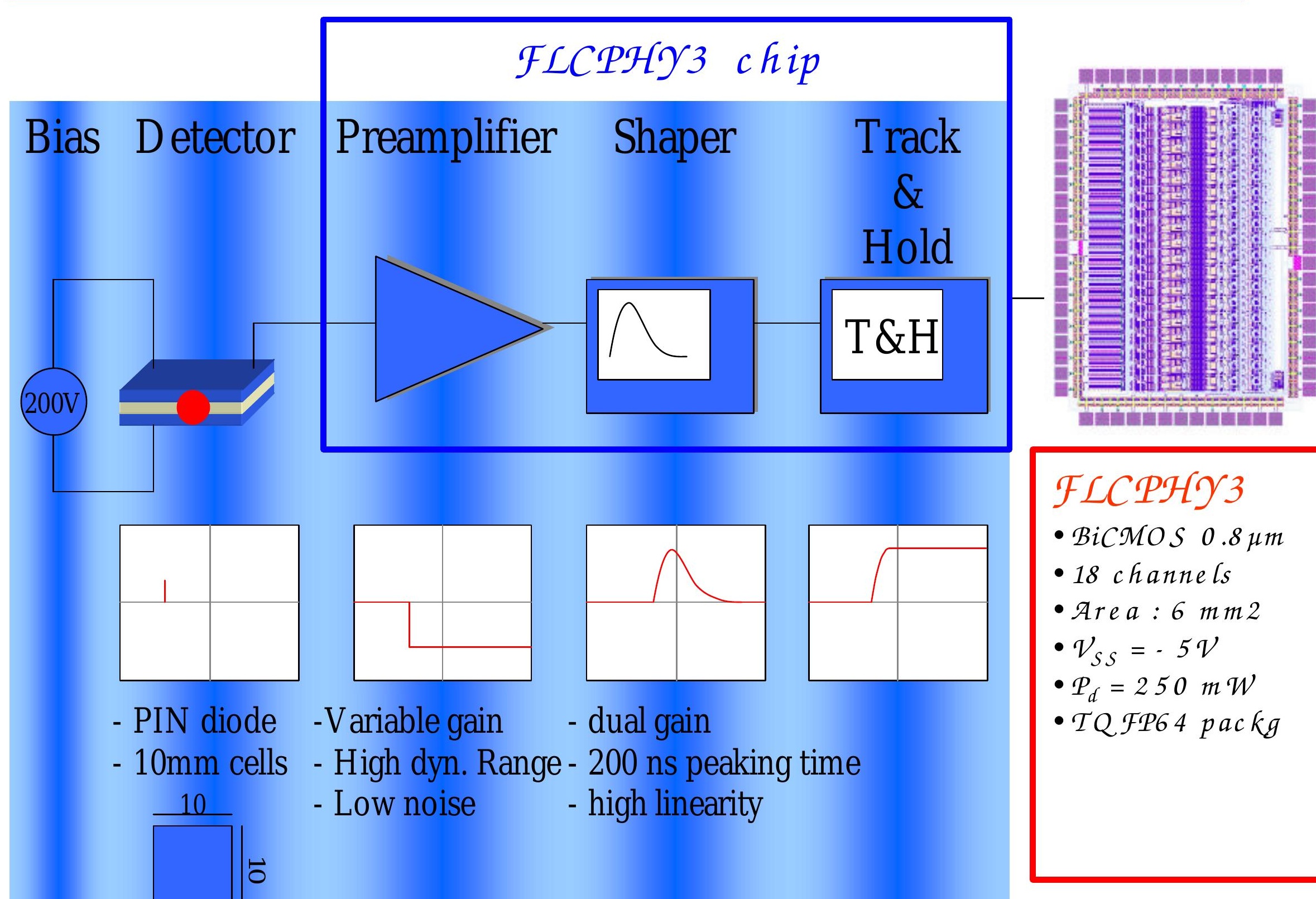




\section{Chip architecture}

- Variable gain preamp $(C f=0.2 \rightarrow 3 p \mathcal{F})$ adapt to severalde tectors

- Dualgain shaper (G1-G10) - > possible studies with larger (166it) dynamic range

- Differential shaper and TrackerHold => better pedestal stability and dispersion

- Multiplexed output : $5 \mathcal{M H z}$

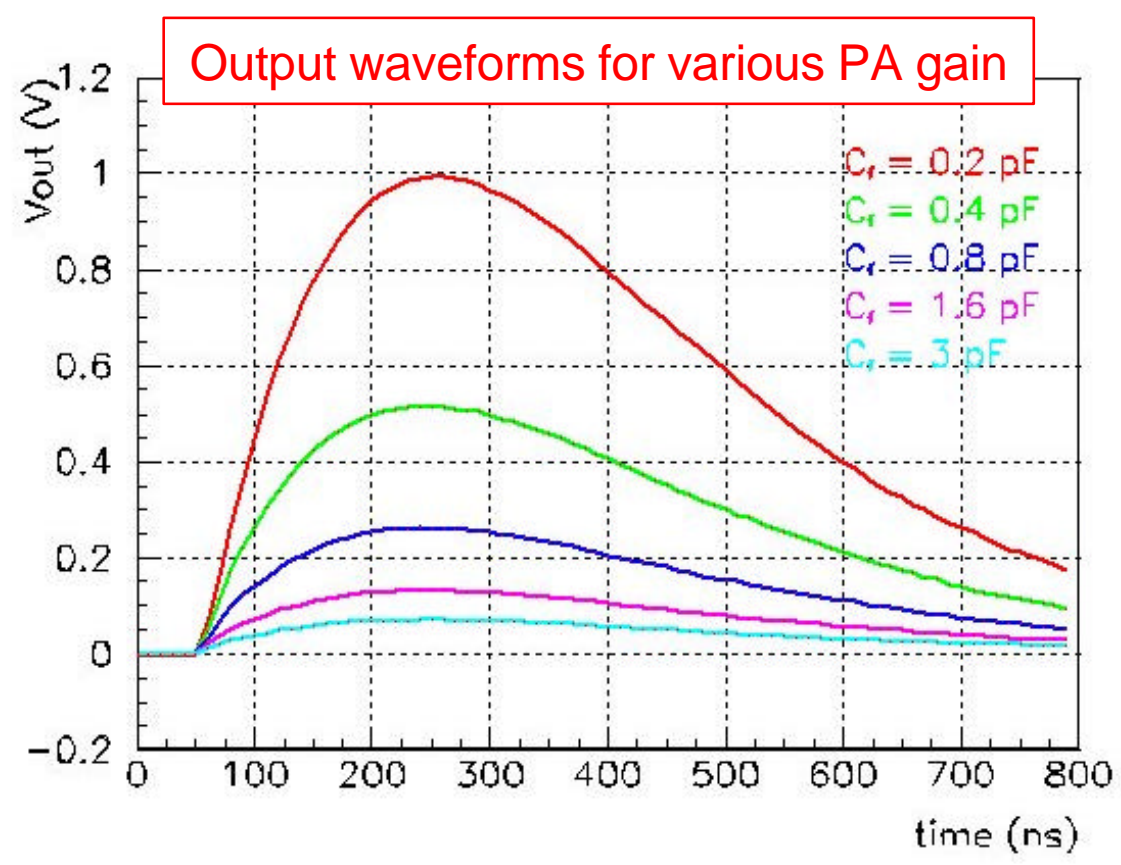

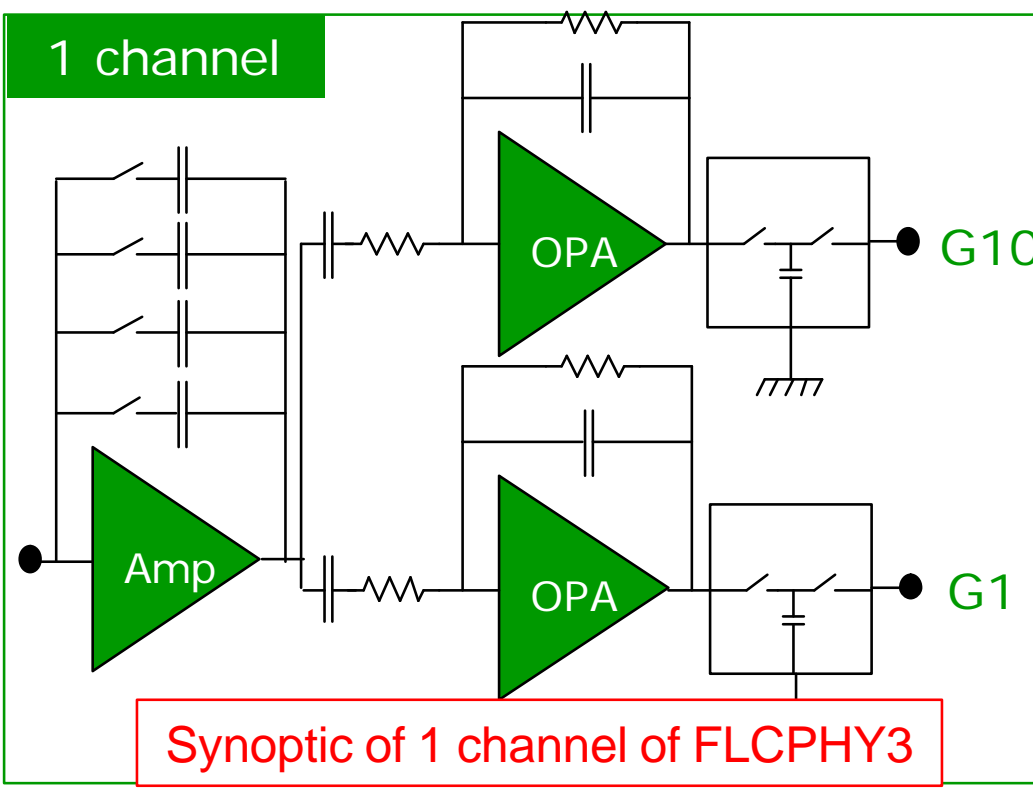

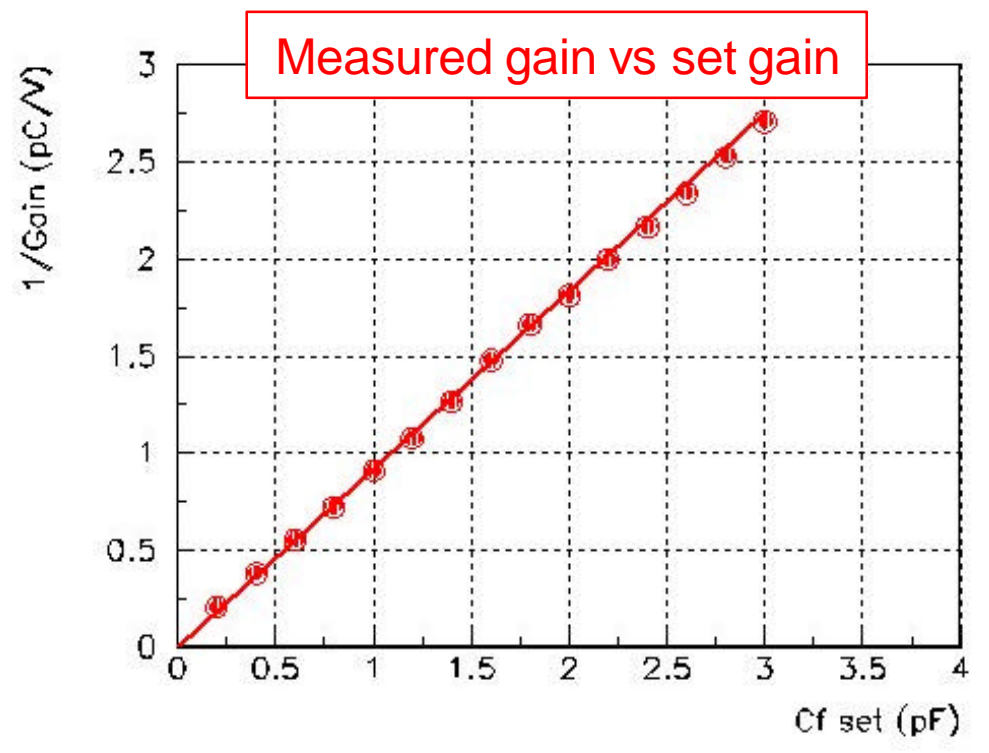


Signal (Gain 1, $\left.C_{f}=1.6 p \mathcal{F}\right)$

- Amplitude $=696 \mathrm{mV} / p C \pm 18 \mathrm{mV}$ $=4.66 \mathrm{mV} / \mathcal{M I P} \pm 2.5 \% \mathrm{rms}$

- Peaking time $=189$ ns \pm 2 ns rms

- Pedestals $=-3.7 \mathcal{V} \pm 4.8 \mathrm{mV} r \mathrm{~ms}$

$\mathcal{N}$ oise

- $\mathcal{C} d=0 p \mathcal{F}: \mathcal{V}_{n}=200 \mu \mathcal{V}$

- $\mathcal{C} d=68 p \mathcal{F}: \mathcal{V} n=410 \mu \mathcal{V}$

Crosstalk : $<0.1 \%$

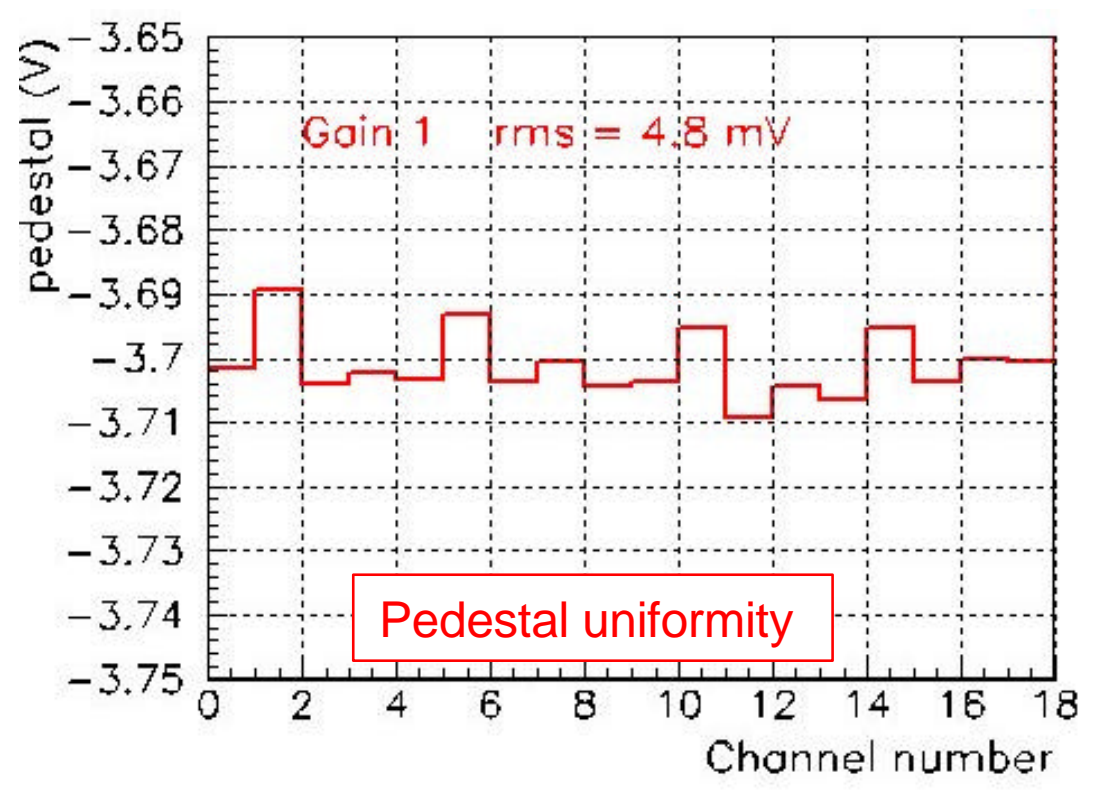

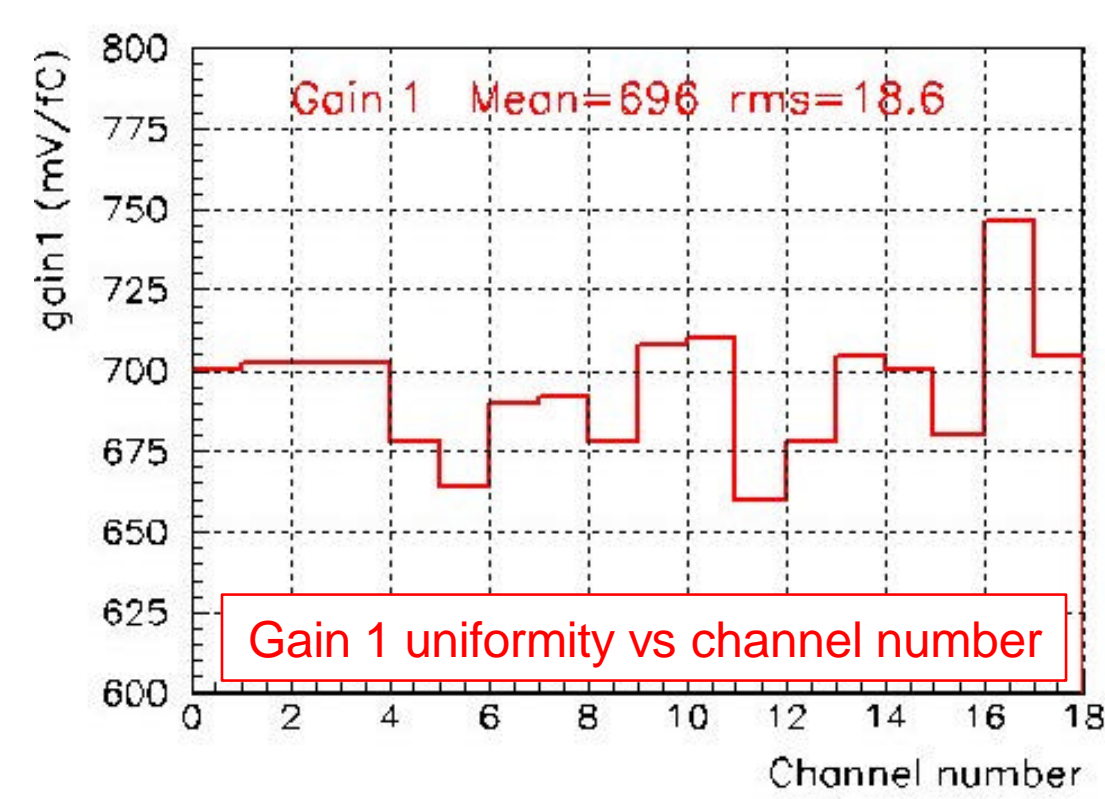

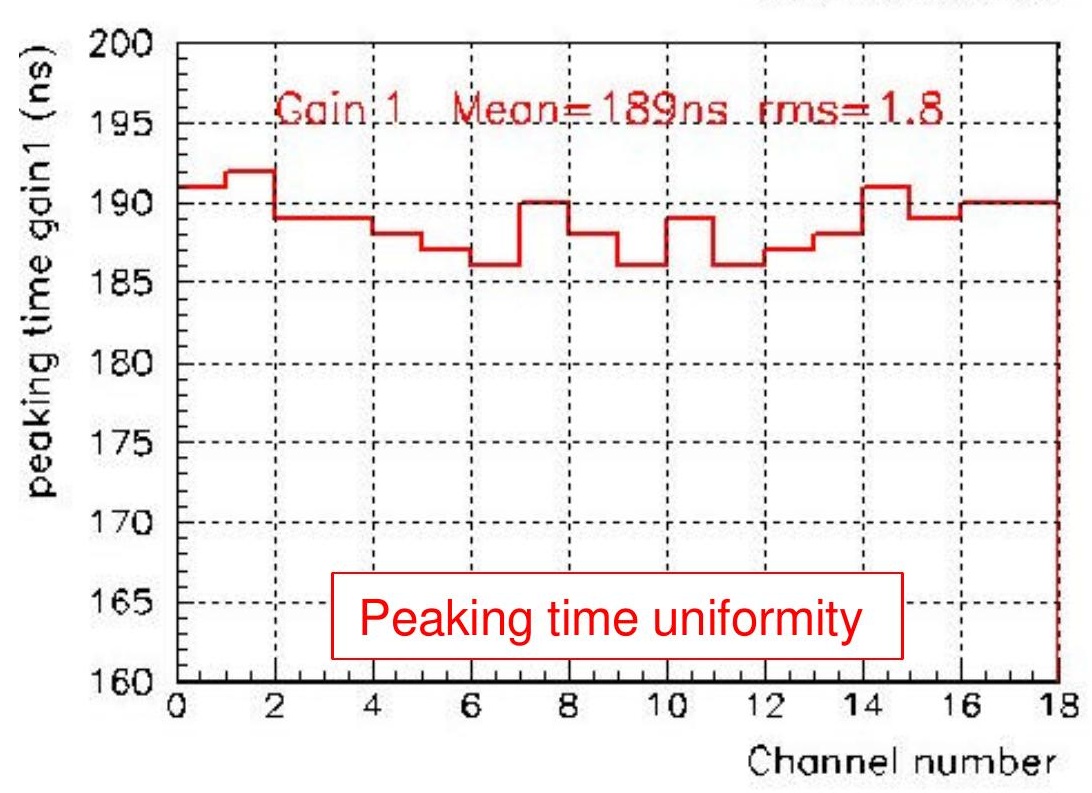


- Signal (Gain 10, Cf $=1.6 p \mathcal{F})$

- Amplitude $=3147 \mathrm{mV} / \mathrm{pC} \pm 94$

- Peaking time $=174 \mathrm{~ns} \pm 2 \mathrm{~ns}$

- Pedestals $=-3.74 \mathcal{V} \pm 8.3 \mathrm{mV} r \mathrm{~ms}$

$\mathcal{N}$ oise

- $\mathcal{C} d=0 p \mathcal{F}: \mathcal{V} n=500 \mu \mathcal{V}$

- $C d=68 p \mathcal{F}: \mathcal{V}_{n}=1.6 \mathrm{mV}$

Crosstalk

口 $<0.2 \%$

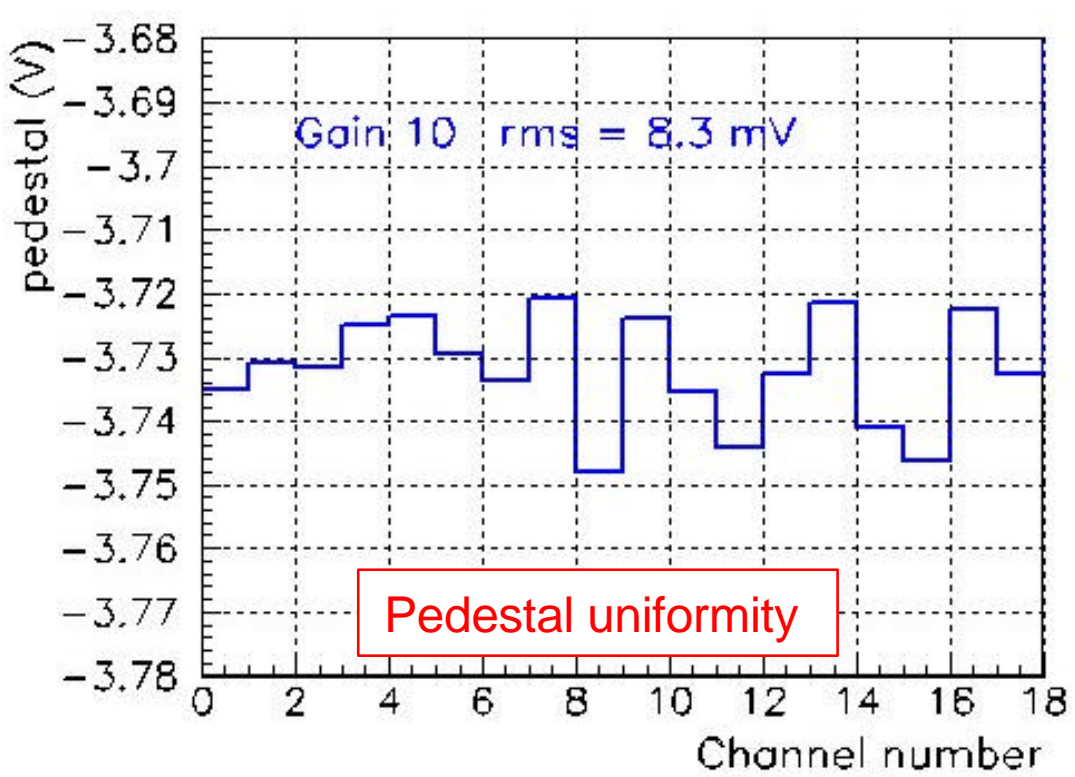

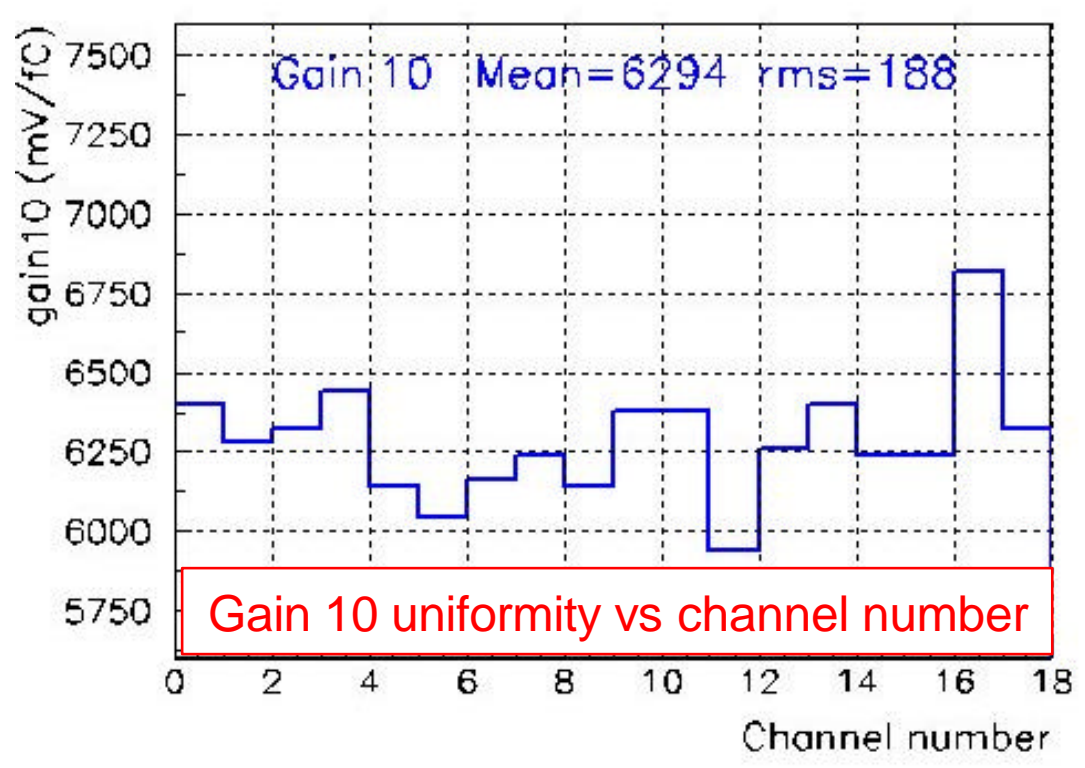

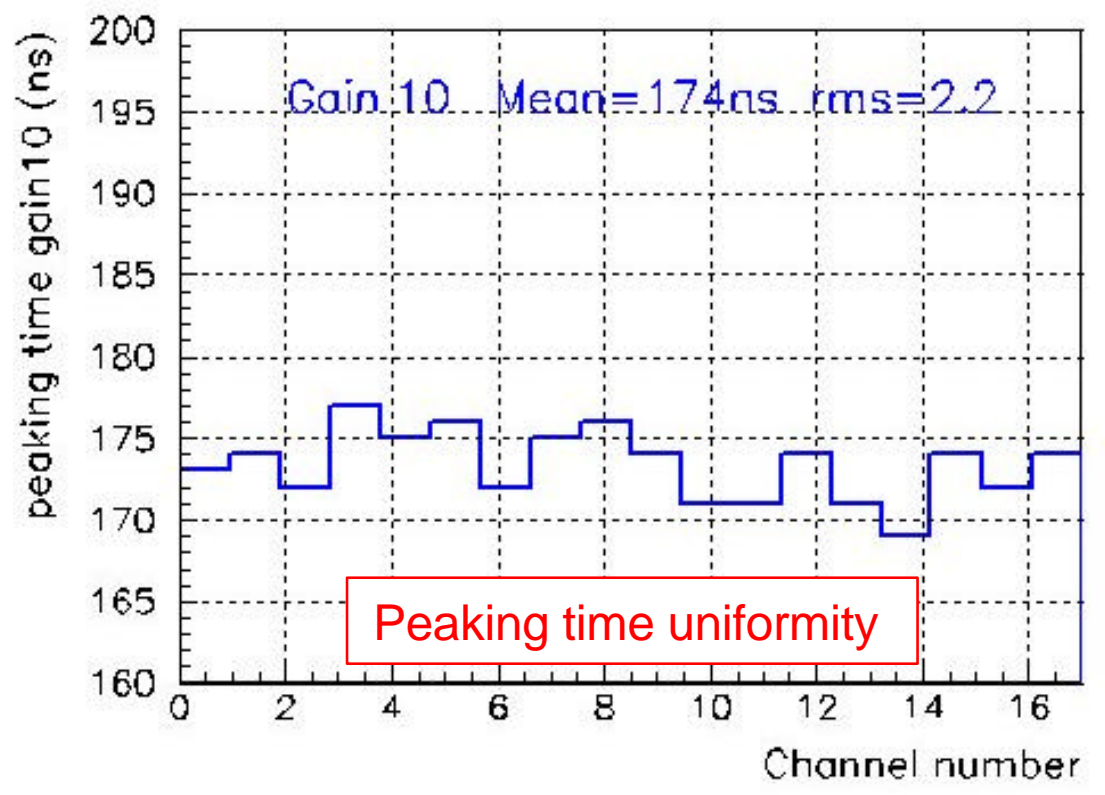


- Measured on all preamp gains

- $C f=0.2,0.4,0.8,1.6,3 p \mathcal{F}$

- Well within $\pm 0.2 \%$

Dynamic range (G1, $\left.C_{f}=1.6 p \mathcal{F}\right)$

- Max output:3 $\mathcal{V}$

- line ar (0.1\%) range : $2.5 \mathrm{~V}$ $=500 \mathcal{M I P S} @ C_{f}=1.6 \mathrm{pF}$

- Noise:

- $200 \mu \mathcal{V}(C d=0)$

- $410 \mu \mathcal{V}(C d=68 p \mathcal{F})$

$-=0.1 \mathcal{M I} \mathcal{P} @ C_{d}=68 \mathrm{pF}$

- Dynamic range : > 12 6its

- 13000 (14 6its) @ $C d=0$

- 6500 (12 6its) @ $C d=68$ pF

- Can be easily extended by using the 6i-gain outputs
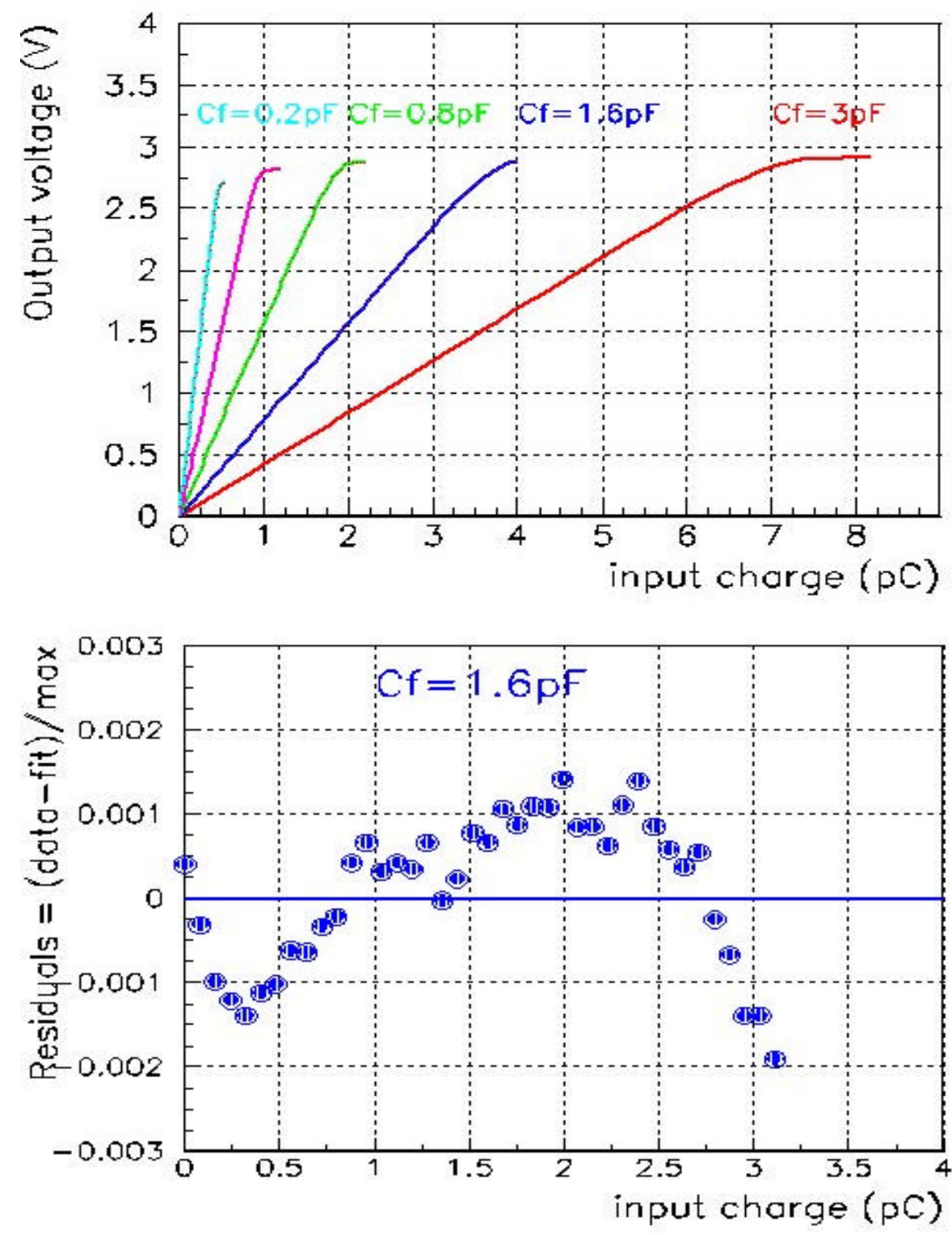
Cosmic test bench at LLR

- $1 M$ MIPinjected in channel 9

- Calculation: $4.97 \mathrm{mV}$

- Measurement: $5.05 \mathrm{mV}$

- Well visible above the noise

- MIP signal with ${ }^{90} S r$ source

- See talkby g.C. Brient

Readout boards

- Developed by aKgroup [P. Dauncey Imperial college]

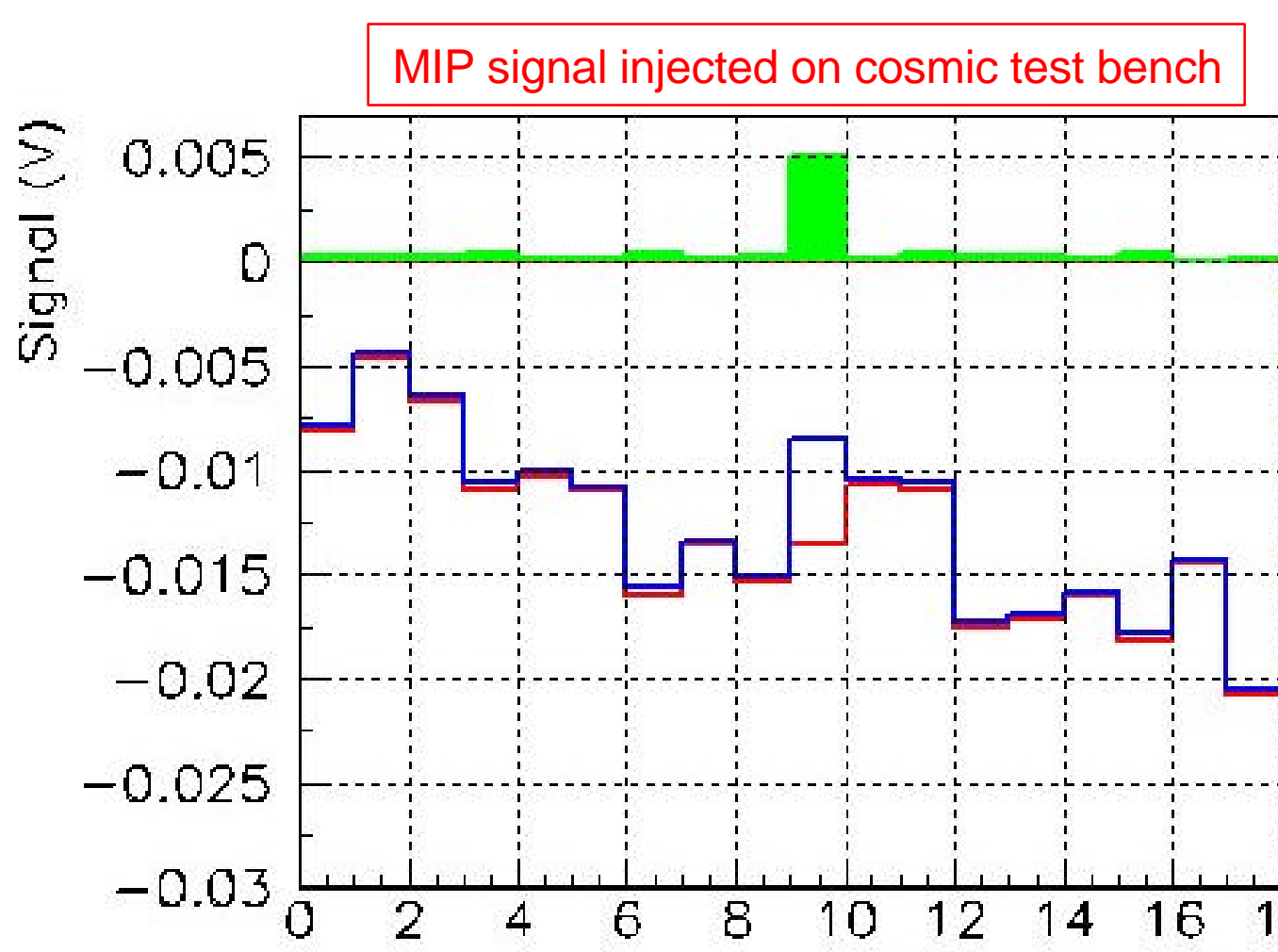

Channel number 
RoD on technological protoptype

- Larger dynamic range : 3000 MIPS (16 6its)

- Lower power : $100 \mu \mathrm{W} / c h$, Autotrigger mode

- See also talk by D. Strom et al.

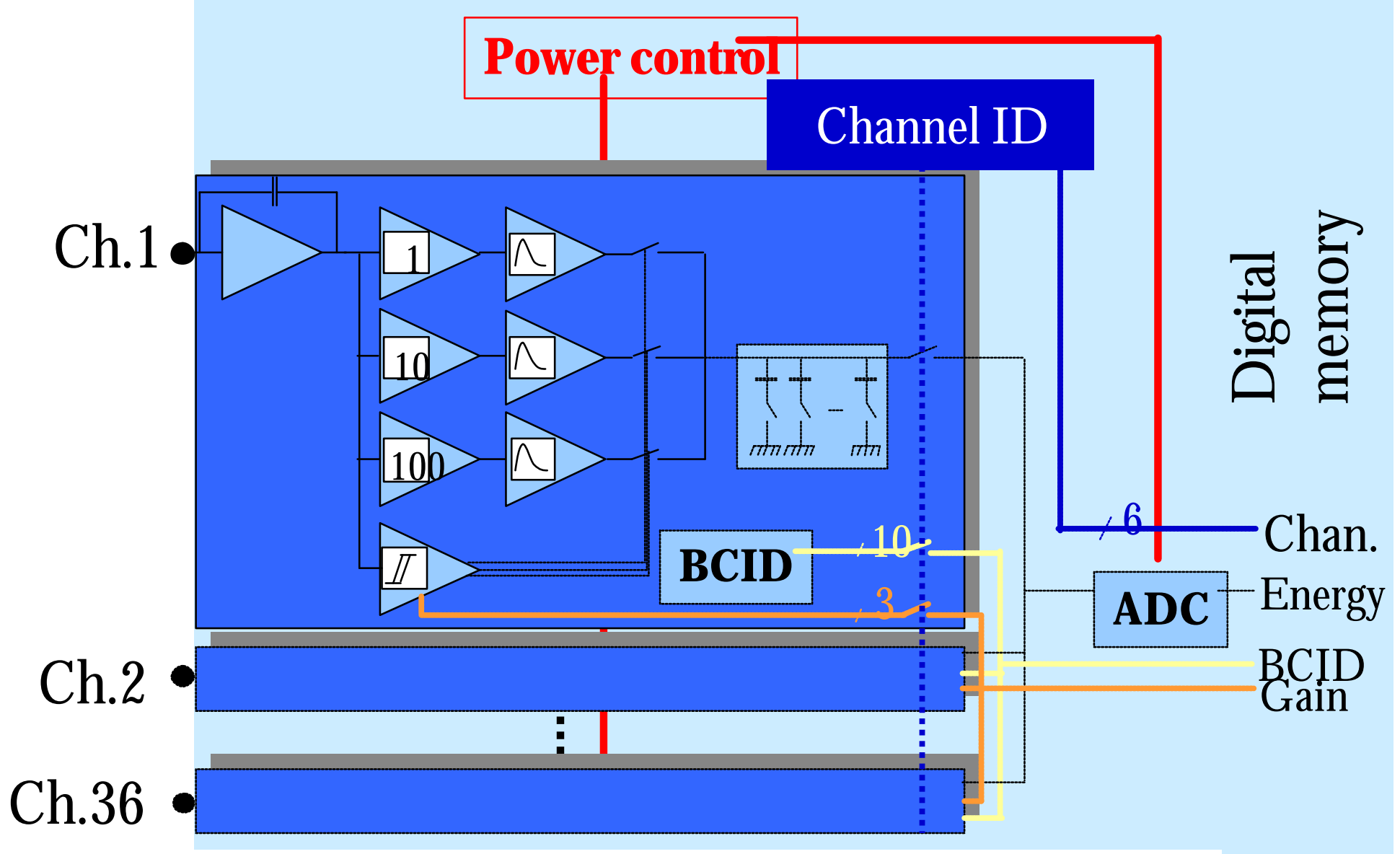


Next steps: technological prototype

Pending questions

- What technology to target for 2010-2020?

- What about signal integrity on a 166 it mixed-signal chip?

- When to digitize? Can we have $1 \mathcal{A D C}$ /channel?

- What (low) power levelcan be reached?
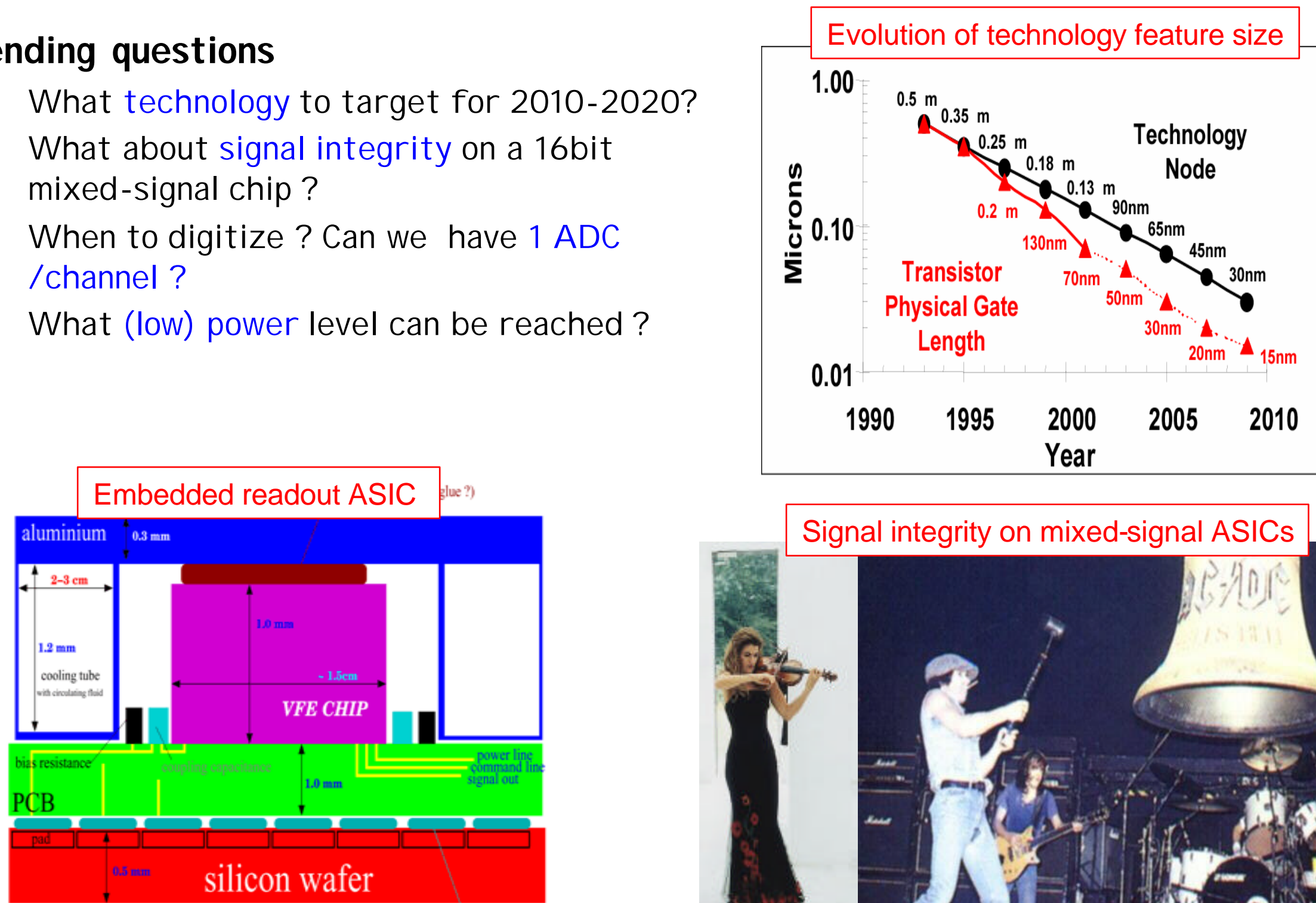

Signal integrity on mixed-signal ASICs

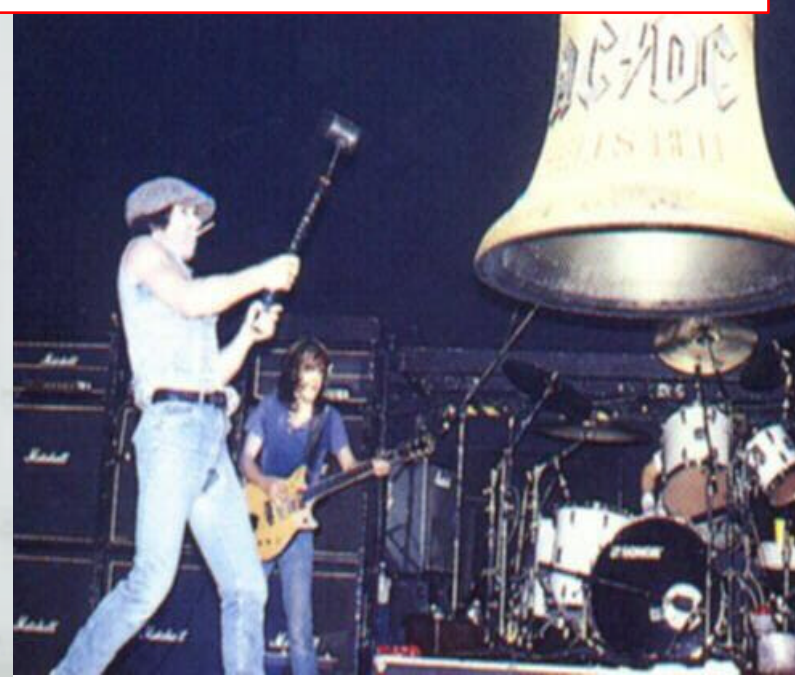


- FLCPHY3 chip fulfills $\mathcal{F} \mathcal{L}$ Wsi testbeam prototype

- Low Noise : 4000e- $=0.1 \mathfrak{M I P}$

- Maximum signal: $600 \mathcal{M I}_{\mathcal{S}}$

- Linearity $: 0.1 \%$, crosstalk $0.1 \%$

- Lowpedestal dispersion: $4.8 \mathrm{mV} r m s=1 \mathfrak{M I P}$

- Canfit other detectors (variable gain 0.2-3pF, bi-gain G1-G10 shaper)

- 1000 chips have been produced for 2004-2005 testbeam

\section{$\mathcal{N e x t}$ steps}

- Low power developments for technological prototype

- Newchip in Sige $0.35 \mu \mathrm{m}$ with Idle mode

- Trying to integrate the $\mathcal{A D C}$...

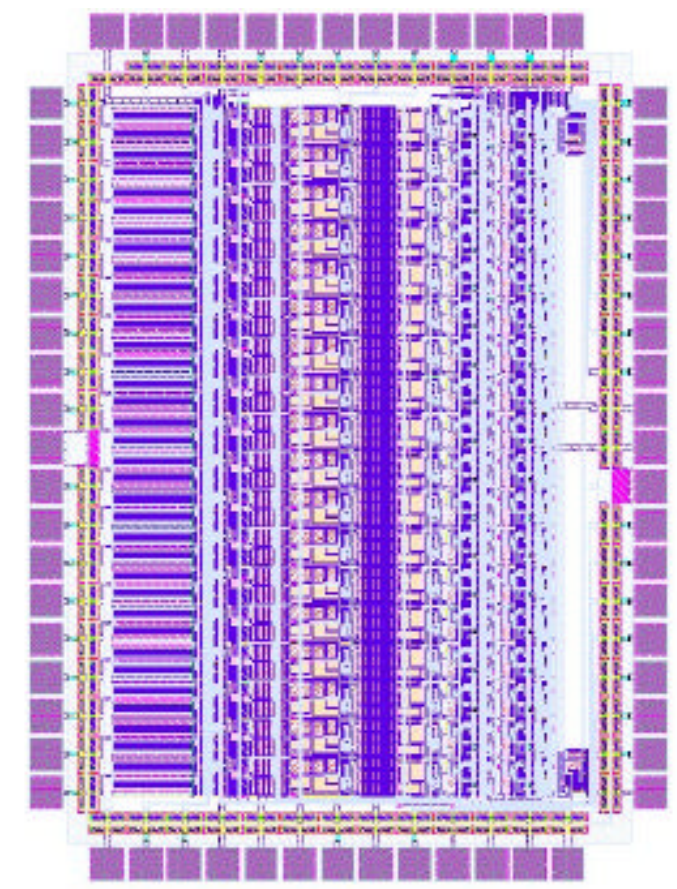

